\title{
Cribriform pattern in brain MRI: A diagnostic clue for mucopolysaccharidoses
}

\author{
S Biswas, MB BS; S Chakraborty, MB BS, MD (Radiodiagnosis)
}

Department of Radiodiagnosis, Institute of Post-Graduate Medical Education and Research, and Seth Sukhlal Karnani Memorial (IPGMEنR and SSKM) Hospital, Kolkata, West Bengal, India

Corresponding author: S Biswas (biswasshamick1@gmail.com)

\begin{abstract}
Mucopolysaccharidoses (MPS) represents a heterogeneous group of inherited lysosomal storage disorders characterised by defective degradation of long-chain complex carbohydrates called glycosoaminoglycans (GAGs). To date, 11 distinct types of MPS have been described, each as a result of deficient enzymatic activity of specific lysosomal hydrolase. The most common types are Hurler and Hunter syndromes. We report a case of a child presenting with macrocephaly, clinically suspected to be due to hydrocephalus. An MRI (3 Tesla) brain study demonstrated the cribriform pattern in the brain caused by dilated perivascular spaces, which is a diagnostic clue for the presence of MPS.
\end{abstract}

S Afr J Rad 2013;17(4):147-148. DOI:10.7196/SAJR.982

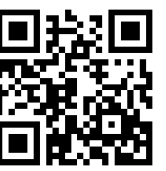

A 2-year-old boy presented with macrocephaly, initially suspected to be due to hydrocephalus. There were no focal neurological deficits. A 3 T MRI of the brain, however, revealed macrocephaly, thickened diploeic spaces (most prominent in the occipital region) and the presence of a J-shaped sella (Fig. 1). A cribriform pattern (otherwise known as 'honeycombing' or 'sieve-like' appearance) (Figs 2 and 3) was present, caused by dilated perivascular spaces, diffusely involving the bilateral subcortical white matter (WM), centrum semiovale, periventricular deep white matter and corpus callosum. Other findings included supratentorial ventriculomegaly, diffuse cerebral cortical atrophy with prominent cortical sulci and extra-axial CSF (cerebrospinal fluid) spaces (Figs 4 and 5). A hypoplastic odontoid process and thickened ligaments posterior to the odontoid, causing craniovertebral (CV) junction stenosis, were also observed (Fig. 1). Skeletal survey of the child revealed features suggestive of mucopolysaccharidosis $1 \mathrm{H}$ (Hurler syndrome), which was further confirmed by quantitative and qualitative urinary glycosoaminoglycan (GAG) analysis ${ }^{[1]}$ revealing elevated levels of dermatan and heparan sulphate.

\section{Discussion}

The earliest and most common abnormality found in patients with MPS is cystic lesions that correspond to enlargement of the Virchow-Robin perivascular spaces (PVS), which are pia-lined spaces that accompany penetrating arteries and arterioles into the brain parenchyma. ${ }^{[2]}$ The PVS dilatation reflects impairment of CSF re-absorption owing to accumulation of GAGs in the vacuolated gargoyle cells ${ }^{[3]}$ of the leptomeninges. With disease progression, the lesions become widespread and extensive, reflecting the development of infarcts in demyelination. ${ }^{[4]}$ The PVS are hypo-intense on T1-weighted images, iso-intense to CSF on T2-weighted images, are suppressed completely on FLAIR images, and do not show any diffusion restriction or contrast enhancement. In MPS, these diffusely enlarged PVS affect the periventricular WM, corpus callosum, basal ganglia, subcortical WM, centrum semiovale, thalami and brain stem, resulting in the cribriform or spindle-like pattern. Such enlargement of the PVS can also be seen in Lowe syndrome ${ }^{[5]}$ and hypomelanosis of Ito. ${ }^{[6]}$ Lacunar infarcts, cystic periventricular leukomalacia, multiple sclerosis, cryptococcosis and neuro-epithelial cysts may also mimic enlarged PVS. ${ }^{[7]}$

Communicating hydrocephalus occurs along with the enlargement of extra-axial subarachnoid spaces. The deposition of GAGs in the meninges impairs the function of arachnoid granulations, leading to abnormal CSF reabsorption. Macrocephaly is a common finding in MPS, often associated with metopic beaking and a scaphocephalic configuration. ${ }^{[2]}$ Diffuse cerebral cortical atrophy may be symmetric or asymmetric, resulting from neuronal death and gliosis, induced by the accumulation of GAGs. Apart from cerebral atrophy, corpus callosum thinning occurs owing to ventricular dilatation and the effect of enlarged PVS within it.

The common posterior fossa abnormalities are mega cisterna magna and arachnoid cysts. Pituitary sella abnormalities include a J-shaped sella - an elongated sella with a shallow anterior convexity owing to an exaggerated normal shallow impression of the sulcus chiasmaticus. Thickening of the diploe also occurs in MPS. Signal intensity abnormalities in the brain parenchyma are commonly seen in the periventricular WM and the basal ganglia. The meninges, most commonly around the craniovertebral (CV) junction, are often thickened and, in severe cases, can compress the medulla or upper cervical cord. Associated atlanto-axial subluxation, odontoid dysplasia and a short posterior arch of atlas can exacerbate the $\mathrm{CV}$ junction stenosis. ${ }^{[2]}$

\section{Conclusion}

Mucopolysaccharidoses (MPS) represent a rare lysosomal storage disorder that can be detected early in the paediatric age group by MRI of the brain. Although the musculoskeletal features are far more characteristic than the neuroradiological abnormalitites, the importance of this cribriform pattern in a paediatric brain lies in the 


\section{CASE REPORT}

fact that it points to the diagnosis of a very few, rare inherited diseases. Consequently, recognition of this pattern can act as an imaging pointer for MPS.

1. Lehman TJA, Miller N, Norquist B, Underhill L, Keutzer J. Diagnosis of the mucopolysaccharidoses.

Rheumatology 2011:50:v41-v48. [http://dx.doi.org/10.1093/rheumatology/ker390]

2. Osborn AG. Osborn's Brain - Imaging, Pathology and Anatomy. 1st ed. Salt Lake City, USA: Amirsys, 2013:887-888.
3. Sequeira FF, Gandhi S, Kini U, Bhat I. Named cells in dermatology. Indian J Dermatol Venereol Leprol 2012;78(2):207-216. [http://dx.doi.org/10.4103/0378-6323.93650]

4. Atlas SW. Magnetic Resonance Imaging of the Brain and Spine. 4th ed. Philadelphia: Lippincott Williams \& Wilkins, 2009:419-420

5. de Carvalho-Neto A. Oculocerebrorenal syndrome of Lowe - magnetic resonance imaging findings in the first six years of life. Arq Neuropsiquiatr 2009;67(2-A):305-307.

6. Almeida AS, Cechin WE, Ferraz J, et al. Hypomelanosis of Ito: Report of a case. J Pediatr (Rio J) 2001;77(1):59-62.

7. Kwee RM, Kwee TC. Virchow-Robin Spaces at MR imaging. Radiographics 2007;27:1071-1086. [http:// dx.doi.org/10.1148/rg.274065722

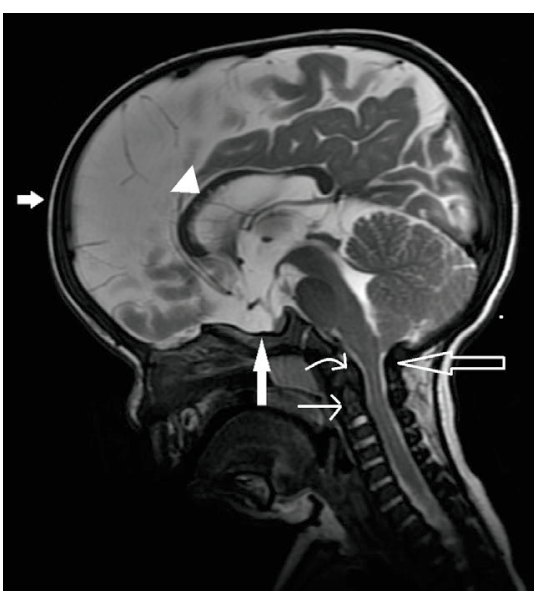

Fig. 1. Sagittal T2 (TR/TE, 3880/109 ms) image shows macrocephaly (small solid arrow) with J-shaped sella (large solid arrow), and dilated perivascular spaces in the corpus callosum (arrowhead). Hypoplastic odontoid process (thin arrow) with thickened ligaments posterior to the odontoid (curved arrow) causing $C-V$ junction stenosis (open arrow) is also noted.

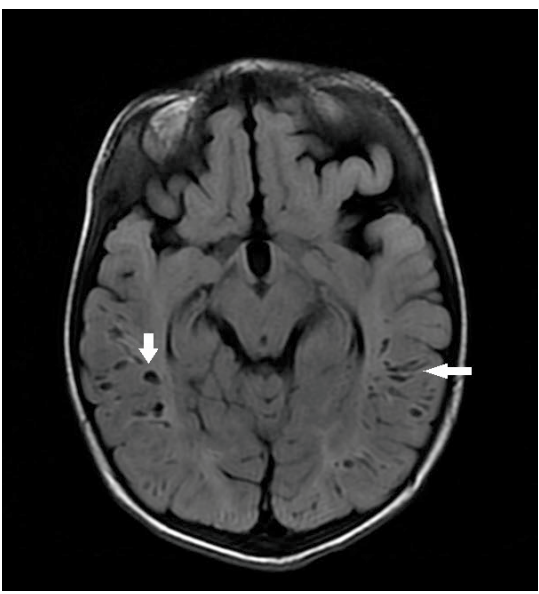

Fig. 3. Axial T2 FLAIR (TR/TE/TI, 9502/125/2250 $m s)$ image at the level of the temporal lobes shows the cribriform pattern (arrows) of enlarged perivascular spaces in the deep white matter.

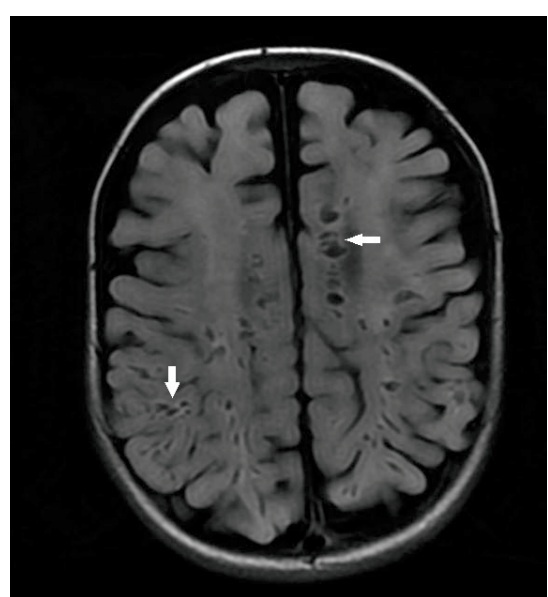

Fig. 2. Axial T2 FLAIR (TR/TE/TI, 9502/125/2250 $m s)$ image at the level of the fronto-parietal lobes shows the cribriform pattern (arrows) of enlarged perivascular spaces in the subcortical and deep white matter and centrum semiovale.

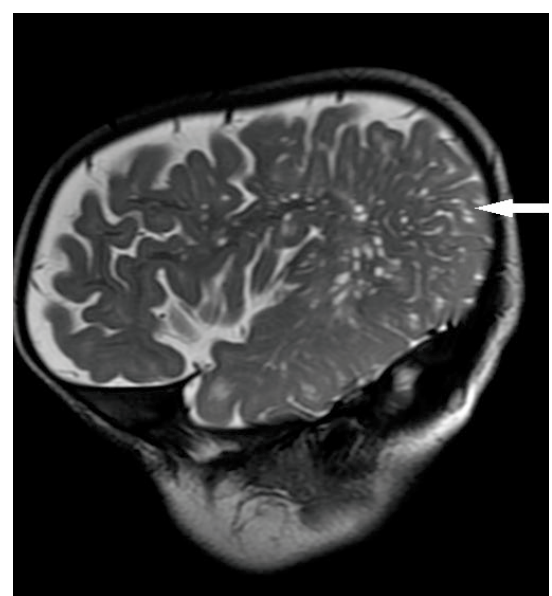

Fig. 4. Sagittal T2 (TR/TE, 3880/109 ms) -weighted image shows the diffusely enlarged perivascular spaces (long arrow).

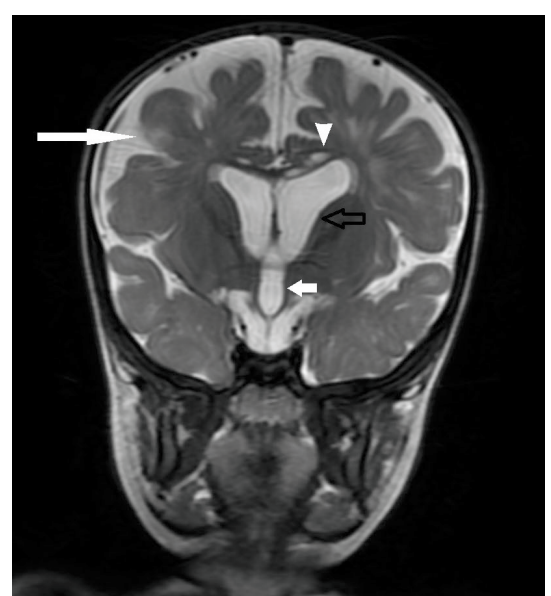

Fig. 5. Coronal T2 (TR/TE, 3260/101 ms)-weighted image shows the hydrocephalus with dilated lateral ventricles (black arrow) and 3rd ventricle (small arrow) with diffuse cerebral cortical atrophy and prominent cortical sulci (long arrow). Also seen is atrophied corpus callosum containing prominent perivascular space (arrowhead) 